\title{
ENVIRONMENTAL MONITORING VIA DRONE
}

\author{
Sutirtha Roy \\ Department of Electrical and Instrumentation Engineering \\ Thapar Institute of Engineering and Technology, Patiala, Punjab, India \\ Geetanshi Gulati \\ Department of Electrical and Instrumentation Engineering \\ Thapar Institute of Engineering and Technology, Patiala, Punjab, India
}

\begin{abstract}
The way of living has evolved tremendously in the last few years. Technological advancement in the field of robotics and Internet of Things (IoT) has not only improved our way of living but also has become an essential part of our lives. Today we can't imagine a worldwithout cars, phones, computers etc. There's still a long way to go and therefore people keep coming up with new innovative ideas. This research was started keeping the same thing in mind. Due to continuous advancements in technology, it is important to monitor the environment in which we live. In this paper we try to present a solution that could be used to continuously monitor the environment via drone.
\end{abstract}

Keywords - Drone, Environment monitoring, Technology,Robotics, Pollution, Accidents, Fire

\section{INTRODUCTION}

Countless are the wonders of science. Technology has completely revolutionized the way we live. There is no aspect of our life in which they do not play a part. Science and technology have conquered time and space. For instance, the modern means of transport have annihilated the natural and geographical barriers separating one country from the other. It has brought all the countries of the world close.

With the advancement in technology and improvement in living standards people strive to develop/invent new products/devices that could make our lives easier. One such field is quadcopters,UAVs; robotics in general.

But not all wonders benefit the universe. These innovations can cause major accidents that take place around the world.

- Air pollution is one of the world's leading factors for death, attributing to 5 million deaths each year.

- It is also becoming one of the leading causes for various kinds of diseases. Air pollution also contributes to about $9 \%$ of total deaths globally.

- Increasing cases of wildfires yearly incurring massivelosses to human, animal life and forest area.
- Fire is the third leading cause of accidental death in home; at least 80 percent of all fire deaths occur in residences.

Considering the above points, we can easily see that there is a need to control environmental pollution which is increasing at an alarming rate and supervise the area in which we live. Hencea combined unit of a drone mounted with a smart environment monitoring system seems quite efficient to reach our goal of reduction of environmental pollution and reduce the risk to life via monitoring the surroundings around us. With the help of the surveillance data provided, we can analyse the status of thatparticular location and take suitable actions as per requirements.

The popularity of quadcopters is increasing day by day due to its wide approach of solving daily life problems. It also has a wide range of industrial applications and helps in assisting manual work. It's a wonder of robotics which can access difficult terrains very easily and help in information acquisition.

Nowadays drones are being used in various sectors due to its low operating and maintenance cost. Since there is an exponential decrease in air quality of our surrounding and due to increase in air pollution, a combination of drone and environmental monitoring module is a need of the hour. A drone can be of various types: Quadcopters, Hexa-copters, Octa-copters etc. Various sensors and cameras can be mounted on the drone for low-cost image and data acquisition.

This paper explains the features, modelling and working of a quadcopter enabled environment monitoring/surveillance system controlled by Arduino Uno microprocessor. The flight controller used here is KK2.1.5 and is controlled by a transmitter-receiver setup. Each and every component has been examined and explained constructively in detail. The main aim is to make it cost effective and also multi-purpose with special emphasis in the field of environment. The drone itself can be used in rescue operations, environmental monitoring, firefighting operations, surveillance, payload delivery and more fields thus making the system quite efficient and flexible. 


\section{International Journal of Engineering Applied Sciences and Technology, 2021 \\ Vol. 5, Issue 9, ISSN No. 2455-2143, Pages 250-253 \\ Published Online January 2021 in IJEAST (http://www.ijeast.com)}

\section{COMPONENTS}

\section{- DRONE FRAME}

This is the frame used in the project. It's made up of 4 plastic fibre arms joined together with a PCB (Printed Circuit Board). It's a Fiberglass PCB and is strong enough to maintain the quadcopters weight.

\section{- BLDC MOTORS}

\begin{abstract}
A brushless DC motor (also known as a BLDC motor or BL motor) is an electronically commutatedDC motor which does not have brushes. The controller provides pulses of current to the motor windings which in turn control the speed and torque of the synchronous motor. Since the motors are brush-less they offer high reliability and longer lifetime to the drone.
\end{abstract}

\section{- Arduino Uno}

Arduino uno is a microcontroller board based on the ATmega328P (datasheet). It has 14 digital input/output pins (of which 6 can be used as PWM outputs), 6 analog inputs, a $16 \mathrm{MHz}$ quartz crystal, a USB connection, a power jack, an ICSP header and a reset button.

It acts as the brain of the drone and all the instructions from the transmitter are first sent to it, processed and then accordingly thrust and direction isgiven to the quadcopter. One main advantage of using Arduino is that we can change the code in it and change the way we want our quadcopter to fly.

\section{- TRANSMITTER \& RECEIVER SET}

A Drone Radio Transmitter is an electronic device that uses radio signals to transmit commands wirelessly via a set radio frequency over to the Receiver, which is connected to the quadcopter being remotely controlled.In other words, it's the device that translates pilot's commands into movement of the multirotor.

A Drone Transmitter transmits instructions via channels. Each channel is an individual action being sent to the quadcopter

Throttle, Yaw, Pitch and Roll are the four main inputs required to control the quad. Each of them uses one channel, so there is a minimum of four channels required.

\section{- Li-Po Battery (Lithium Polymer Battery)}

Lithium polymer batteries, more commonly known asLiPo, have high energy density, high discharge rate and light weight which makes them a great componentfor energy providers in drones.

\section{- Propellers}

Drone Propellers are the key component that keeps the multirotor in the air, they have the most direct impact on how the drone flies, and over the lifetime of the multirotor they are likely to add up to be the biggest investment that one has made to keep flying. They are also going to be the component that is damaged and replaced the most often.

\section{- $\quad$ Electric Speed Controller}

An Electronic Speed Controller (ESC) is a device that regulates the power of an electric motor, allowing it to throttle from $0 \%$ to $100 \%$. It ensures that all four motors of the quadcopters are moving according to thecommands given by the transmitter.

\section{- FLIGHT CONTROLLER}

A flight controller is a hardware typically in the form of a PCB. It is a compact device embedded with memory, processor, sensors etc.

In our case we are using flight controller KK 2.1.5.

It is a multi-rotor flight controller. In this we get an option of auto level as well. It's lightweight which gives it an edge over others. It has inbuilt functions so no need of programming it, if required one can reprogram it as per their needs.

KK 2.1.5 is a board containing ATMEL mega 664PA,8-bit AVR RISC based microcontroller with $64 \mathrm{~K}$ of memory. It's quite user friendly and has firmware pre-defined in it. While activating or deactivating the board there is an audio warning fromthe piezo buzzer of KK 2.1.5. It is the most stable board because it has an inbuilt gyroscope, in the form of $6050 \mathrm{MPU}$, and auto level function. This board haseight motor outputs, five control inputs, an LCD display, polarity protected voltage sensor input, an ISP header, six-axis accelerometer/gyroscope, a fuse protected piezo output. The user-defined signals fromthe flight controller board or KK board are processed by ATMEL 644PA IC and these control signals arepassed to the ESC's installed on the frame of the drone.

\section{- DHT22}

The DHT22 is a Temperature and humiditysensor. The sensor is easy to interface with microcontrollers. DHT22 contains a thermistor which is used to measure dry bulb temperature and humidity sensor measured by utilizing changes in the conductivity of the substrate material that issensitive to moisture.

The sensor can measure temperature from $-40^{\circ} \mathrm{C}$ to $80^{\circ} \mathrm{C}$ and humidity from $0 \%$ to100\% with an accuracy of $\pm 1^{\circ} \mathrm{C}$ and $\pm 1 \%$. 


\section{International Journal of Engineering Applied Sciences and Technology, 2021 \\ Vol. 5, Issue 9, ISSN No. 2455-2143, Pages 250-253 \\ Published Online January 2021 in IJEAST (http://www.ijeast.com)}

\section{- MQ2}

MQ2 gas sensor is an electronic sensor used for sensing the concentration of gases in the air such as LPG, propane, methane, hydrogen, alcohol, smoke and carbon monoxide. MQ2 sensor is commonly used for safety purposes. It is easy to use and, highly reliable and low in cost.

Front view

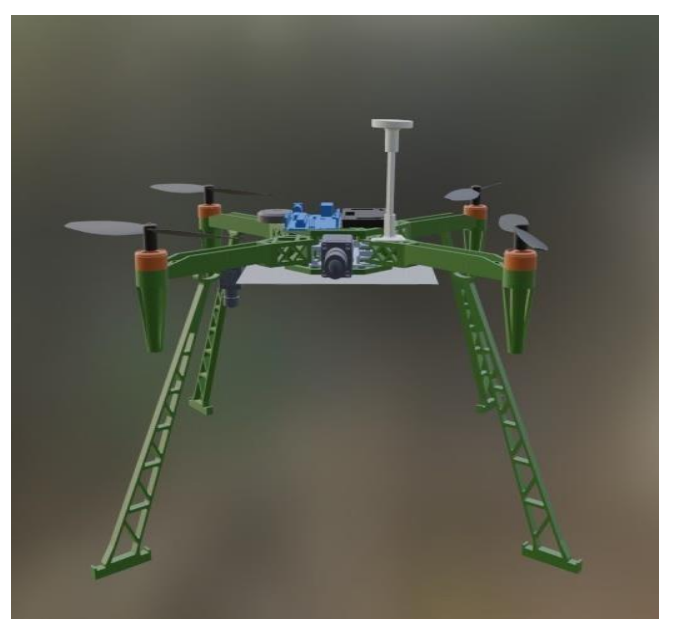

Side View

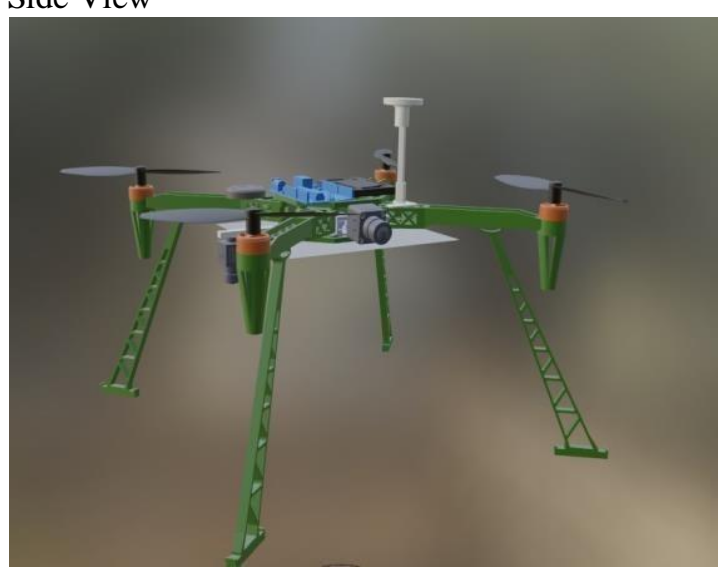

\section{PROPOSED MODEL}

\section{A. ALGORITHM}

Each drone will be mounted with an environmental monitoring module. It will take the surrounding data via various calibrated sensors and then the collected data will be sent to the ground station for the analysis purpose. The step-by-step procedure to function this whole proposed idea:-

1) Surveillance of the environment via an environmental monitoring unit mounted on a drone. This drone is controlled via a receiver transmitter module.
2) Capturing of the surrounding environmental parameter data using various calibrated sensors.

3) Using the DHT22 sensor for collecting temperature and humidity readings of the surrounding and we are using MQ2 sensor for collecting air quality related readings of the surrounding.

4) Using Digi Xbee-PRO 900HP RF module for sending the collected data to the control station for the analysis of the collected environment related data of the examined surroundings.

5) The transmitted signal is collected by the receiver module of the Xbee which is present in the control station end. Later on, this collected data is examined via LABVIEW software. This data can be used further for the extensive research, survey or analysis as per user's requirements.

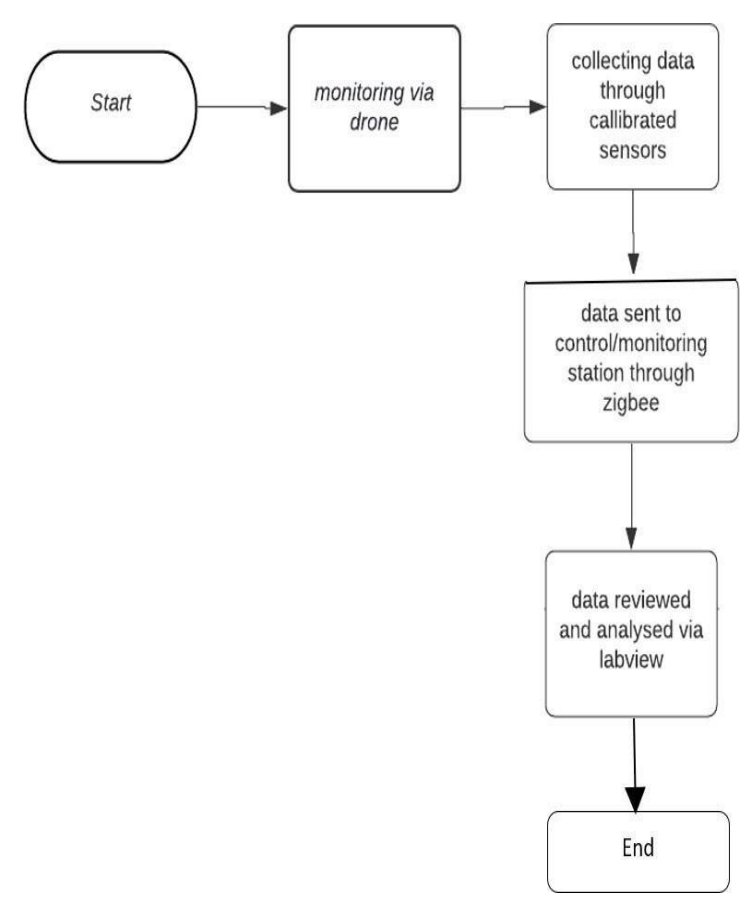

B. WORKING

1. Calibrated sensors are used for this wireless module.(sensors used are smoke sensor, humidity temperature sensor). ZigBee module used for the wireless communication purpose. Extensive modulecalibrated under the XCTU software.

2. After calibration of various sensors and the Zigbee(XBee) with the computer/ any electronic display unit. We start our working module.

3. All the data is collected from the sensors and via calibrated sensors it is sent to Arduino UNO.

4. From Arduino UNO the data gets translated to the computerized calibrated ZigBee transmitterreceivermodule. 


\section{International Journal of Engineering Applied Sciences and Technology, 2021 \\ Vol. 5, Issue 9, ISSN No. 2455-2143, Pages 250-253 \\ Published Online January 2021 in IJEAST (http://www.ijeast.com)}

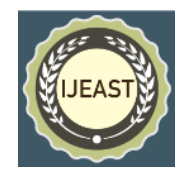

5. The receiver end module accepts the incoming pulsedmodulated signal from the transmitter end.

6. After receiving the signal, it gets converted into waveforms denoting various aspects like smoke level, temperature, etc., on the display screen and this graphical representation is done by the LabVIEW software of National Instruments.

7. Live data monitoring using the Classic LabVIEW software, an amazing innovative software made byNational Instruments.

\section{CONCLUSION}

As we are already aware of the fact that rising air pollution and death due to fire are some major issues of concern and need to be taken into account immediately. Therefore, the model proposed in this paper aims at solving these problems by monitoring the environment quickly and efficiently. By this we could be able to monitor the environment which is the need of the hour especially at places like schools, colleges, railway stations, metro stations, markets, shopping centres etc. With this we are not only preventing the damage that could be caused but protecting the lives as well.

\section{FUTURE SCOPE}

Since the technology keeps evolving, there's a lot of scope of improvement and development ahead. We plan to make our drone "intelligent" by making it autonomous and giving it thepower to analyse the environment itself and warn us only when it's something serious.

These autonomous UAVs can also be accompanied by other similar drones that aid in firefighting response over a larger affected area. For communication between these drones, we can take the help of ArduPilot and the sensor data from these devices is collected via the XBee Pro modules through a "cluster network", where we have a single PAN Coordinator which here represents the base station and different drones in a cluster act as Full Function Devices(routers) which further relay information to Reduced Function Devices (sensors, controllers, activators, etc.)

This model will work on neural networks which will be trained on the data collected from the environment. Also, a swarm of drones would be effective in future monitoring purposes.

\section{REFERENCE}

[1] Forbes, P.B.C. (2015). "Chapter 1: Perspectives on the Monitoring of Air Pollutants". In Barcelo, D. (ed.). Monitoring of Air Pollutants: Sampling, Sample Preparation and Analytical Techniques.

Comprehensive Analytical Chemistry. 70. Elsevier. pp. 3-9. ISBN 9780444635532.

[2] Hu, J.; Lanzon, A. (2018). "An innovative tri-rotor drone and associated distributed aerial drone swarm control". Robotics and Autonomous Systems. 103: 162-174.

[3] Sharma, Abhishek; Basnayaka, Chathuranga M.Wijerathna; Jayakody, Dushantha Nalin K. (May 2020)."Communication and networking technologies for UAVs: A survey". Journal of Network and Computer Applications.

[4] Carlson, Daniel F.; Rysgaard, Søren (1 January 2018). "Adapting open-source drone autopilots for real-time iceberg observations". MethodsX. 5: 1059-1072..

[5] Tice, Brian P. (Spring 1991). "Unmanned AerialVehicles

- The Force Multiplier of the 1990s". Airpower Journal. Archived from the original on 24 July 2009. Retrieved 6 June 2013.

[6] Ugurlu, Measuring the Impact of Virtual Instrumentation for Teaching and Research. In: IEEE Global Engineering Education Conference (EDUCON), pp. 152-158 (2011)

[7] Portillo, E., Cabanes, I., Marcos, M., Orive, D., Sanchez, J.A.: Design of a VirtualInstrumentation System for a Machining Process. IEEE Transactions on Instrumentation and Measurement 56(6), 26162622 (2007)

[8] O. Edewede, D. Jazani and G. Epiphaniou, "Internet of Things Forensics: Challenges and approaches", Proc. of 9th International Conference on Collaborative Computing: Networking Applications and Worksharing (CollaborateCom), 2013.

[9] Kim, S. \& Paulos, E. (April 2010). inAir:Sharing indoor air quality measurements and visualizations. Earth, Wind, Flyer. 81-84.

[10] Fensterstock, J. C.; Kurtzweg, J. A.; Ozolins, G. (1971). "Reduction of Air Pollution Potential through Environmental Planning". Journal of the Air Pollution Control Association. 21 (7): 395-399.

[11] Gauderman, W (2007). "Effect of exposure to traffic on lung development from 10 to 18 years of age: a cohort study". The Lancet. 369 (9561): 571-77

[12] Bruck (2001) The who, what, where and why of waking tofire alarms. A review, Fire safety journal 36, p. 623-639. 